\title{
Ultrathin $\beta$-tellurium layers grown on highly oriented pyrolytic graphite by molecular-beam epitaxy
}

\author{
Jinglei Chen ${ }^{\mathrm{a}, \dagger}$, Yawei Dai ${ }^{\mathrm{a}, \dagger}$, Yaqiang Ma ${ }^{\mathrm{b}}$, Xianqi Dai ${ }^{\mathrm{b},{ }^{*}}$, Wingkin $\mathrm{Ho}^{\mathrm{a}}$, Maohai Xie ${ }^{\mathrm{a},{ }^{*}}$

\begin{abstract}
Monolayer Tellurium (Te) or tellurene has been suggested by a recent theory as a new two-dimensional (2D) system with great electronic and optoelectronic promises. Here we present an experimental study of epitaxial Te deposited on highly oriented pyrolytic graphite (HOPG) by molecular-beam epitaxy. Scanning tunneling microscopy of ultrathin layers of Te reveals rectangular surface cells with the cell size consistent with the theoretically predicted $\beta$-tellurene, whereas for thicker films, the cell size is more consistent with that of the [10 $\overline{1} 0]$ surface of the bulk Te crystal. Scanning tunneling spectroscopy measurements show the films are semiconductors with the energy bandgaps decreasing with
\end{abstract} \\ increasing film thickness, and the gap narrowing occurs predominantly at the valance-band maximum (VBM). The latter is understood by strong \\ coupling of states at the VBM but a weak coupling at conduction band minimum (CBM) as revealed by density functional theory calculations.
}

\section{Introduction}

Two-dimensional (2D) materials have been the subject of intensive research in recent years. They exhibit an array of interesting properties as well as application promises in future nano-electronic, optoelectronic, spin and valley electronic devices. ${ }^{1-10}$ Examples of the widely studied 2D systems include graphene, ${ }^{1-3}$ silicene, ${ }^{4}$ phosphorene, ${ }^{5}$ borophene, ${ }^{6,7}$ stanene ${ }^{8}$ and transition-metal dichalcogenides, ${ }^{9}, 10$ covering a wide range of properties from, e.g., metals, semiconductors to insulators. Efforts are continuing to add more members to the 2D family. A recent theoretical study has suggested a group- $\mathrm{VI}$ element, i.e., tellurium ( $\mathrm{Te}$ ) that can stabilize in the 'monolayer' form. The tellurene as is called consists of a trilayer of Te atoms arranged in one of three configurations: 1T$\mathrm{MoS}_{2}$-like $(\alpha-\mathrm{Te})$, tetragonal ( $\beta$-Te), and $2 \mathrm{H}-\mathrm{MoS}_{2}$-like $(\gamma$-Te). The first two were found semiconducting with energy gaps of $0.75 \mathrm{eV}$ and $1.47 \mathrm{eV}$, respectively, which are appealing for microelectronic and optoelectronic applications. ${ }^{11}$

At ambient conditions, crystalline tellurium (Te-l) in the bulk form is a semiconductor with a narrow band gap of $0.34 \mathrm{eV}$. It has a helical structure with the space group $\mathrm{P} 3_{1} 21$ or $\mathrm{P} 3_{2} 21$ depending on the chirality of Te helical chains along the $c$-axis. Atoms in each helical chain are covalently bonded whereas the chains themselves are held by van der Waals (vdW) forces and packed into a hexagonal lattice. ${ }^{12}$ This would make it possible to fabricate Te nanostructures, for example, Te nanowires and nanotubes that were reportedly obtained by syntheses using different methods and techniques. ${ }^{13-20}$ However, Te thin films particularly in the ultrathin and monolayer limit remain to be documented. At the time of preparation of this manuscript, we became aware of a recent study of epitaxial $\mathrm{Te}$ on graphene/6H-SiC(0001) by using molecular beam epitaxy (MBE). ${ }^{21}$ It was asserted that the film was a bulk-truncate of Te crystal along the [1010] direction, which could only form on graphene but not on highly oriented pyrolytic graphite (HOPG). In this paper, we report growth of epitaxial Te on HOPG by MBE where the epifilm shows a surface lattice constant that is consistent with the theoretically predicted $\beta$-tellurene for ultrathin films. By a scanning tunnelling microscopy and spectroscopy (STM/STS) study, we establish that the films are semiconductors and the bandgap changes with film thickness, and this change happens predominantly at the valence-band maximum (VBM), the position of conduction-band minimum (CBM) remains almost constant. Aided by density functional theory (DFT) calculations, we attribute the latter to a strong coupling of states at the valence band edge, which has the character of the $p$-orbital of the top layer Te in the tellurene tri-layer. Interestingly, as the epifilm thickness increases, we note a change of surface cell size from $4.26 \times 5.42 \AA^{2}$ to $4.42 \times 5.93 \AA^{2}$ that are consistent with theoretically predicted $\beta$-tellurene and bulk Te lattice, respectively, indicating a possible phase transformation between these two phases.

\section{Experimental}

\section{MBE growth and STM measurement}

Te deposition on a freshly cleaved and thoroughly annealed HOPG substrate was carried out in an Omicron MBE reactor having the base pressure of $\sim 2 \times 10^{-10} \mathrm{mbar}$. The flux of Te was generated from a standard Knudsen cell operated at 540 $\mathrm{K}$. The substrate temperature was held constant at $400 \mathrm{~K}$ during deposition. Reflection high-energy electron diffraction (RHEED) operated at $15 \mathrm{keV}$ was employed to monitor the sample surface during the growth process and the streaky diffraction pattern signifies atomically smooth surfaces of the grown film and is affirmed by STM measurements at roomtemperature. Atomic resolution STM and STS measurements were carried out using a Unisoku STM system. Constantcurrent mode was used for all STM measurements. For STS, the lock-in technique was employed using a modulation voltage of $15 \mathrm{mV}$ and frequency of $991 \mathrm{~Hz}$.

\section{DFT calculation}

Calculations were performed based on the DFT in conjunction with the projector-augmented wave potentials ${ }^{22}$ as implemented in the Vienna $A b$ initio Simulation Package (VASP). ${ }^{23,24}$ The exchange-correlation potential was described through the Predew-Burke-Ernzerhof functional within the generalized gradient approximation formalism ${ }^{25}$. A plane wave basis set with a cutoff energy of $520 \mathrm{eV}$ and K-mesh of 0.02 determined by a fine grid of Monkhorst-Pack method ${ }^{26}$ in the 
Brillouin zone was found to produce good converged results. The atomic structures were relaxed using conjugate gradient algorithm until the forces on all unconstrained atoms were smaller than $0.005 \mathrm{eV} / \AA \AA$. A vacuum layer of $20 \AA$ along the $z$ direction was constructed to eliminate the interaction with spurious replica images. ${ }^{27}$ The zero damping DFT-D3 method of $\mathrm{Gimme}^{28}$ was used to account for long range vdW interaction between layers. (a)

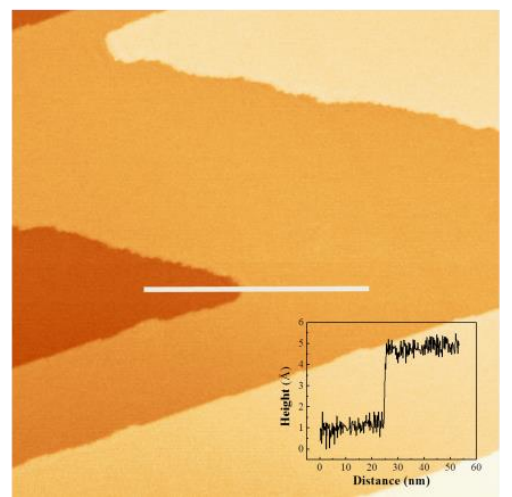

(b)

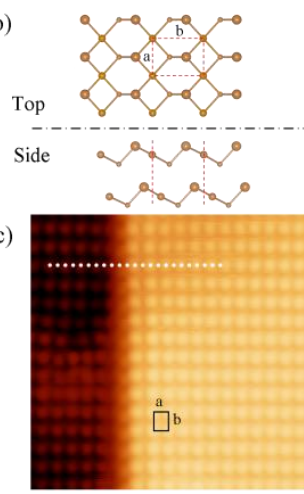
Side
Fig. 1 (a) Topographic image (size: $100 \times 100 \mathrm{~nm}^{2}$, sample bias: $1 \mathrm{~V}$ ) of an epitaxial Te film showing an atomically flat terraces separated by steps of height of $\sim 4 \AA$. The inset presents a line profile taken along the white line drawn in the image. (b) A stick-andball model of $\beta$-tellurene layer viewed from top and side, respectively. The dotted lines mark the surface unit cell. (c) Atomic resolution STM image (size: $8 \times 8 \mathrm{~nm}^{2}$, bias: $0.6 \mathrm{~V}$ ) showing rectangular lattices as highlighted by the black rectangle.

\section{Results and discussion}

We note firstly that the deposit is of pure Te according to Auger electron spectroscopy measurements (refer to Supplementary). The grown Te films show atomically flat terraces by STM and affirmed also by the streaky RHEED patterns. Fig. 1a shows a topographic STM image of an asgrown sample for a nominal thickness of $\sim 20 \AA$. Large terraces delineated by atomic steps are clearly seen. Line profile measurements (inset) reveal step heights of $4.0 \pm 0.2 \AA$. Atomic resolution image (Fig. 1c) of such a surface reveals rectangular lattice instead of the hexagonal one of the substrate and the in-plane lattice constants are measured to be $a=4.26 \AA$ and $b=5.42 \AA$. They are consistent with the theoretically predicted $\beta$-tellurene that is schematically drawn in Fig. 1b. This differs from the report of Ref. ${ }^{21}$, where a larger lattice of $4.42 \times 5.93 \AA^{2}$ was noted for the Te layer grown on graphene. The latter is more in agreement with that of a bulk truncate of Te-I crystal along [10 10$]$. We remark that a similarly larger lattice was also measured in our experiments from a thicker Te films (refer to Supplementary). Therefore, there could be a phase transformation from $\beta$-tellurene to Te bulk crystal as the film thickness increases. Further studies are however still needed to establish the thickness dependence of the phases as suggested by theory. ${ }^{11}$

The STS measurements taken at flat terraces of the Te film are shown in Fig. 2a for different thicknesses. The latter were established by measuring the domain heights relative to the exposed substrate (see Supplementary). As seen, there is a clear density-of-state (DOS) gap, confirming that Te epifilms are semiconductors. The Fermi level (OV) is found below the mid-gap energy, suggesting the sample may be hole-doped. The origin of hole doping can be from intrinsic defects, such as vacancies, in sample. ${ }^{29,30}$ We however do not have sufficient data to correlate the Fermi energy shift with defects in sample. An alternative effect could be due to charge transfer between HOPG substrate and the ultrathin Te layer. In any case, the experimental STS appear to agree with the DFT calculated DOS shown in Fig. $2 b$ of $\beta$-Te. In particular, the electronic gap of epitaxial Te is film-thickness dependent, e.g., $\sim 0.65 \mathrm{eV}$ for the 5 tri-layer film (black line in Fig. 2a), but $\sim 0.58 \mathrm{eV}$ and $0.53 \mathrm{eV}$ for the 6 and 7 tri-layers (red and blue lines). The DFT calculations reveal similar change (Fig. 2b), though the magnitude differs. Another interesting feature seen from Fig. $2 a$ is that the energy gap change happens predominantly at the VBM. The CBM stays almost constant, reflecting the very character of states at the VBM and CBM of ultrathin Te layers.
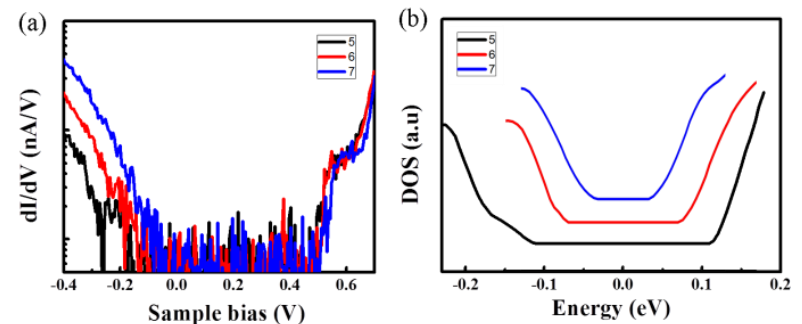

Fig. 2 (a) STS of epitaxial Te of different thicknesses (black, red and blue lines are for 5 , 6 and 7 trilayers, respectively). (b) DFT-calculated DOS of 5, 6 and 7-trilayer $\beta$-Te. The Fermi level $(\mathrm{OV})$ is set in middle of the gap. Curves are vertically shifted for clarity.

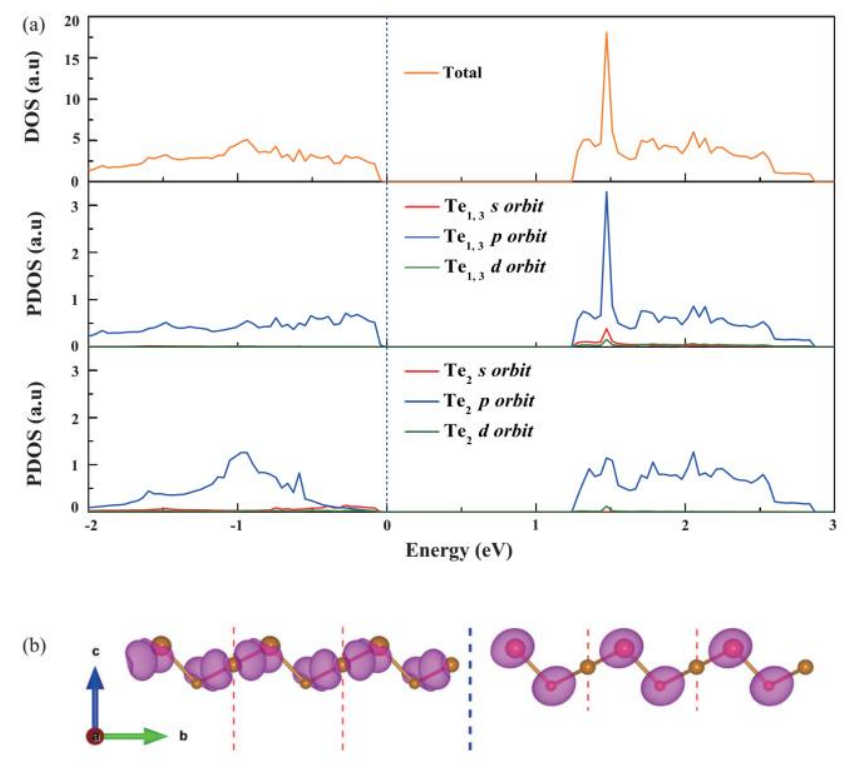

Fig. 3 (a) DFT calculated PDOS, where $\mathrm{Te}_{1,3}$ refer to atoms in the top and bottom layer of Te-tellurene, $\mathrm{Te}_{2}$ refers that in the middle layer. (b) Spatial distribution for states at the CBM (left) and VBM (right) for a single $\beta$-Te trilayer. The isosurface is at $0.04 \mathrm{e}^{-3}$. 
To gain further insight on the states at VBM and CBM, we carried out the DFT calculations for the partial charge densities of $\beta$-Te under different layer thicknesses. Fig. 3(a) presents the derived partial density of states (PDOS) and Fig. 3(b) plots the spatial distribution of charge densities at the energies of CBM and VBM for a single $\beta$-Te tri-layer (refer to Supplementary for the double and triple tri-layer cases). It can be seen that the VBM states are mainly of the $p$-orbitals of the top and bottom layer Te atoms in the $\beta$-Te tri-layer, whereas the CBM states are mainly of the middle layer Te and thus confined within the $\beta$-Te tri-layer. As a result, stacking $\beta$-Te into multi-layer films will lead to inter-layer coupling of the VBM states but less influence on the CBM states. Consequently, one expects much more pronounced changes from the VBM than CBM as the film thickness varies, which agrees with the experiment. As a comparison, the partial charge density distribution of helical Te shows the different characteristics (see Supplementary). So the observation of the VBM change as the film thickness varies may have lent an additional support for the $\beta$-Te rather than a bulk-truncate film in our experiment.

We have further acquired the STS across a step separating a 5layer from a 6-layer terrace of epitaxial Te. The result is summarized in Fig. 4a. As seen, apart from a discontinuous jump in energy bandgap at the step, corresponding to the thickness-dependent bandgap change as discussed above, no other obvious feature can be discerned. There is no apparent band bending, although we could not rule out a band-bending effect over a shorter spatial range detectable by STM. A similar observation has also been made by Guo and co-workers, where no band bending was seen across Te steps, despite an obvious bending observed at steps separating the substrate and monolayer Te islands. ${ }^{21}$

(a)
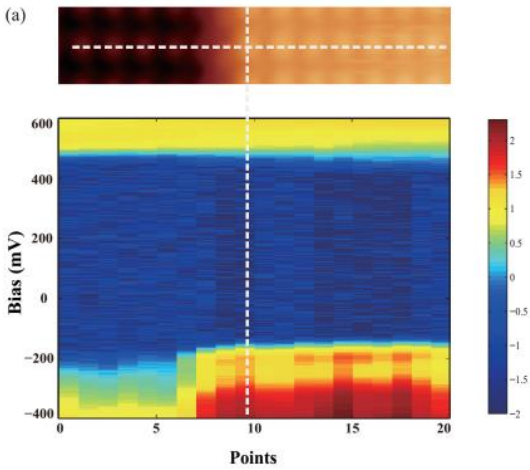

Fig. 4 (a) Differential conductance spectra presented in the logarithm intensity map (bottom) taken across a 5-to-6 layer step (i.e., the horizontal dashed line in the STM image shown on top and the white dotted line drawn in Fig. 1c). (b) A spectrum taken over the step edge marked by the vertical dashed line in (a).

One might expect the presence of edge states at steps due to broken translational symmetry and if so there could be a Fermi level pinning, resulting in the band-bending as seen, for example, at monolayer-to-bilayer steps of $\mathrm{MoSe}_{2}{ }^{31,}{ }^{32}$ However, our STS measurements at Te film steps reveal no obvious mid-gap edge state (see Fig. 4b). STM measurements (e.g., Fig. 1c) reveal no reconstruction of step-edge atoms or apparent atom relaxation, at least within the limit of resolution of our experiment.

Finally, we would like to comment on the growth behaviour of Te on HOPG, which has made it difficult to obtain monolayer Te in this experiment. There appeared to be a high propensity of the epifilm to grow in mounds of multilayer high, leaving some areas of the substrate exposed (see Supplementary). This behaviour of growth resembles the Volmer-Weber growth mode. However, we note the mounds often to take the wedding-cake like morphology with flat tops and terraces at the sides. At the bottom of the mounds, we occasionally discern small terraces of monolayer and bilayer Te, but the areas of such mono- or bi-layer terraces are too small and deep relative to the mound tops that accurate and reliable STS measurements are not possible. Change of substrate may be needed in order to achieve large scale tellurene layers.

\section{Conclusions}

We have grown Te films on HOPG by MBE and the in-plane lattice symmetry and constant conform to the theoretically predicted $\beta$-Te for ultrathin layers. STS measurements reveal semiconducting properties of the Te epilayers and the energy bandgap decreases with increasing layer thickness. The gap variation occurs predominantly at the VBM, which can be attributed to the different characters of the VBM versus CBM states as revealed by our DFT calculations. We observe no obvious band-bending at steps.

\section{Acknowledgements}

This work was financially supported in part by a Collaborative Research Fund (HKU9/CRF/13G) sponsored by the Research Grants Council (RGC), Hong Kong Special Administrative Region and by the National Natural Science Foundation of China (Grant nos. 61674053).

\section{References}

1 K. S. Novoselov, A. K. Geim, S. V. Morozov, D. Jiang, Y. Zhang, S. V. Dubonos, I. V. Grigorieva and A. A. Firsov, Science, 2004, 306, 666-669.

2 C. Berger, Z. Song, X. Li, X. Wu, N. Brown, C. Naud, D. Mayou, T. Li, J. Hass and A. N. Marchenkov, Science, 2006, 312, 11911196.

3 A. C. Neto, F. Guinea, N. M. Peres, K. S. Novoselov and A. K. Geim, Reviews of modern physics, 2009, 81, 109.

4 P. Vogt, P. De Padova, C. Quaresima, J. Avila, E. Frantzeskakis, M. C. Asensio, A. Resta, B. Ealet and G. Le Lay, Physical review letters, 2012, 108, 155501.

5 L. Li, Y. Yu, G. J. Ye, Q. Ge, X. Ou, H. Wu, D. Feng, X. H. Chen and Y. Zhang, Nature nanotechnology, 2014, 9, 372-377.

6 A. J. Mannix, X.-F. Zhou, B. Kiraly, J. D. Wood, D. Alducin, B. D. Myers, X. Liu, B. L. Fisher, U. Santiago and J. R. Guest, Science, 2015, 350, 1513-1516.

7 B. Feng, J. Zhang, Q. Zhong, W. Li, S. Li, H. Li, P. Cheng, S. Meng, L. Chen and K. Wu, Nature chemistry, 2016.

8 F.-f. Zhu, W.-j. Chen, Y. Xu, C.-I. Gao, D.-d. Guan, C.-h. Liu, D. Qian, S.-C. Zhang and J.-f. Jia, Nature materials, 2015, 14, 1020-1025. 
9 B. Radisavljevic, A. Radenovic, J. Brivio, i. V. Giacometti and A. Kis, Nature nanotechnology, 2011, 6, 147-150.

10 X. Xu, W. Yao, D. Xiao and T. F. Heinz, Nature Physics, 2014, 10, 343-350.

11 Z. Zhu, X. Cai, C. Niu, S. Yi, Z. Guo, F. Liu, J.-H. Cho, Y. Jia and Z. Zhang, ArXiv, 2017, 1701.08875.

12 F. A. Blum and B. C. Deaton, Physical Review, 1965, 137, A1410-A1417.

13 Q. Wang, M. Safdar, K. Xu, M. Mirza, Z. Wang and J. He, ACS nano, 2014, 8, 7497-7505.

14 Y. Xia, P. Yang, Y. Sun, Y. Wu, B. Mayers, B. Gates, Y. Yin, F. Kim and H. Yan, Advanced materials, 2003, 15, 353-389.

15 B. Mayers and Y. Xia, Advanced materials, 2002, 14, 279-282.

16 M. Mo, J. Zeng, X. Liu, W. Yu, S. Zhang and Y. Qian, Advanced materials, 2002, 14, 1658-1662.

17 Y. J. Zhu, W. W. Wang, R. J. Qi and X. L. Hu, Angewandte Chemie, 2004, 116, 1434-1438.

18 C. J. Hawley, B. R. Beatty, G. Chen and J. E. Spanier, Crystal Growth \& Design, 2012, 12, 2789-2793.

19 M. Safdar, X. Zhan, M. Niu, M. Mirza, Q. Zhao, Z. Wang, J. Zhang, L. Sun and J. He, Nanotechnology, 2013, 24, 185705.

20 G. a. Tai, B. Zhou and W. Guo, The Journal of Physical Chemistry C, 2008, 112, 11314-11318.

21 X. Huang, J. Guan, B. Liu, S. Xing, W. Wang and J. Guo, Arxiv, 2017, 1703.07062.

22 G. Kresse and D. Joubert, Physical Review B, 1999, 59, 1758.

23 G. Kresse and J. Furthmüller, Comp Mater Sci, 1996, 6, 15-50.

24 P. E. Blöchl, Physical review B, 1994, 50, 17953.

25 J. P. Perdew, K. Burke and M. Ernzerhof, Physical review letters, 1996, 77, 3865

26 H. J. Monkhorst and J. D. Pack, Physical review B, 1976, 13, 5188.

27 A. Bermudez, F. Jelezko, M. Plenio and A. Retzker, Physical review letters, 2011, 107, 150503.

28 S. Grimme, J. Antony, S. Ehrlich and H. Krieg, The Journal of chemical physics, 2010, 132, 154104.

29 A. Kolobov, Journal of non-crystalline solids, 1996, 198, 728731.

30 M. Kastner and H. Fritzsche, Philosophical Magazine Part B, 1978, 37, 199-215.

31 C. Zhang, A. Johnson, C.-L. Hsu, L.-J. Li and C.-K. Shih, Nano letters, 2014, 14, 2443-2447.

32 H. Liu, H. Zheng, F. Yang, L. Jiao, J. Chen, W. Ho, C. Gao, J. Jia and M. Xie, ACS nano, 2015, 9, 6619-6625. 